\title{
Using of artificial neural networks to assess the residual resource of trunk pipelines
}

\author{
Ilya Lapiga $^{1 *}$, Andrey Shchipachev ${ }^{1}$, and Dmitriy Osadchiy ${ }^{2}$ \\ ${ }^{1}$ Saint Petersburg Mining University, Department of Transportation and storage oil and gas, 2, 21st Line, St Petersburg 199106, Russia \\ ${ }^{2}$ Saint Petersburg Mining University, Department of Development and exploitation of oil and gas fields, 2, 21st Line, St Petersburg \\ 199106, Russia
}

\begin{abstract}
A large number of oil and gas pipelines in the Russian Federation have been in operation for over 20 years. For these pipelines, the issue of assessing the residual resource is relevant. Today, much attention is paid to the problem of long-term durability of pipelines. Trunk pipelines are under the influence of cyclic loads and influences arising during operation. The acting stresses in the pipe wall do not exceed the allowable ones, however, they cause micro-damage to the metal structure. When assessing the cyclic fatigue of a metal, the main criterion is the relative damage to the metal. The use of non-destructive testing methods (ultrasonic and magnetic), as well as the establishment of a relationship between the number of cycles and diagnostic parameters, will improve the accuracy of the residual life assessment. When analyzing several diagnostic parameters, the question of data interconnection becomes relevant. Since establishing an empirical or semi-empirical relationship between ultrasonic and magnetic properties is a complex task, artificial neural networks (ANNs) can be used to solve this problem. The use of ANN in the diagnostics of trunk pipelines will increase the accuracy of the assessment and eliminate the subjectivity of data interpretation.
\end{abstract}

\section{Introduction}

Russia has one of the longest pipeline networks in the world. The length of the main pipelines is over 250 thousand kilometers [1]. At the same time, the age of many of them exceeded the standard operating life or is close to this. In such conditions, the problem of assessing the residual life of such pipelines becomes particularly relevant. During their diagnosis, according to the regulatory and technical documentation available today, much attention is paid to the presence of defects, as the main dangerous factor that can cause a major accident.

Evaluation of the technical condition is a calculation of the stress-strain state of the pipeline wall, which is under the influence of a number of factors such as internal pressure, ambient temperature, seismic impact, etc. [2]. The obtained evaluation result should be evaluated by the criterion of the limiting state of the structure, ie compared with the limit state for this type of structure under given operating conditions [3]. Currently, in the field of diagnostics and assessment of the residual life of structures operating under variable load conditions, an approach to determining the technical condition based on an assessment of long-term strength is increasingly being used.

A similar approach can be applied to main pipelines, since oil and gas pipelines also operate under conditions of variable loads arising during their operation.

\section{The Impact of cyclic loads on the strength of the metal}

The danger of cyclic loads arising is that the destruction of structures becomes possible under the action of stresses not exceeding the tensile strength for given conditions. With a sufficiently large number of cycles (section II), destruction can occur under the influence of a random load that may occur during operation of the pipeline (seismic, vibration, etc.). If destruction did not occur in section II, then the strength passes over time to section III, where destruction can occur under the action of workloads.

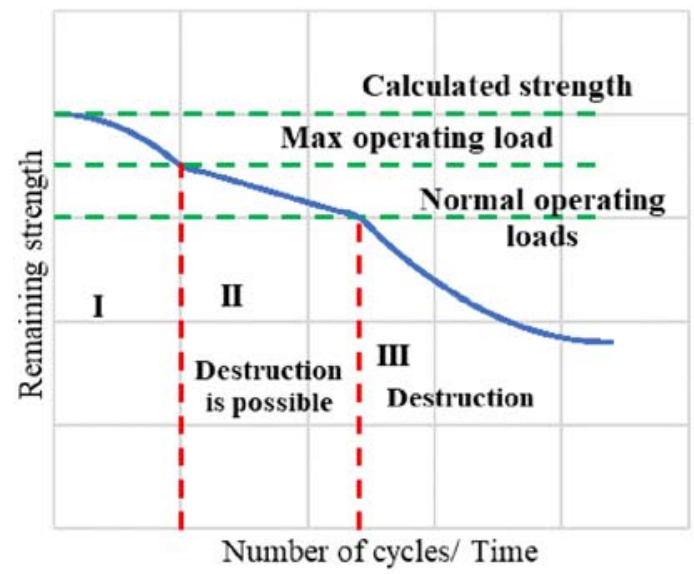

Fig.1. Curve of remaining strength.

* Corresponding author: irlapiga@gmail.com 
Figure 2 shows the surface images of 09Mn2Si steel operated under cyclic loading conditions. The left part shows the initial stage of microcrack nucleation. Cracks are localized, have a small size. As the number of cycles increases, the number of cracks grows, their density increases. Ultimately, a large number of microcracks are combined into a network of cracks. Directly from such a network, a macrocrack can subsequently form, which can be detected with the naked eye.

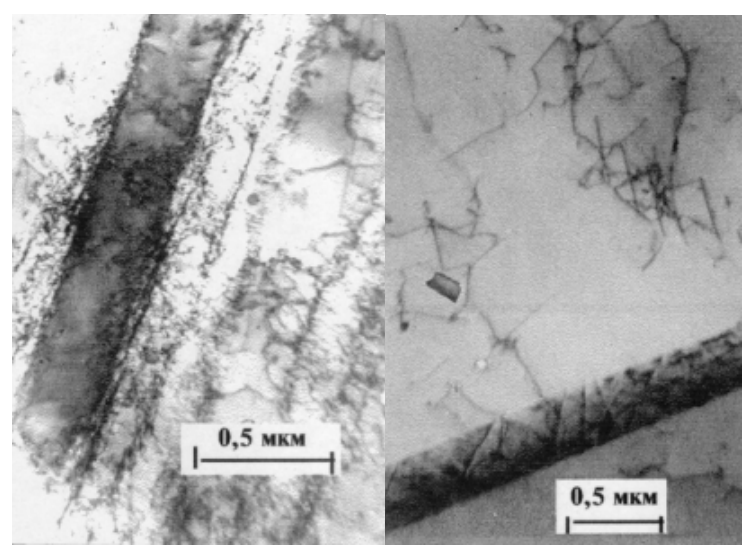

Fig.2. Microcracks of steel during fatigue process.

\subsection{Cyclic loads in main oil pipelines}

Cyclic loads arising in oil pipelines relate to low-cycle loading of the structure.

Loads arise mainly for technological reasons, which in the vast majority of cases are starting or stopping pumping units. Also, one of the reasons may be the switching of valves. The main factor under these cyclic loads is the increase in oil pressure in the pipeline. According to the regulatory documentation, cyclic loading will include all registered by the pipeline control system pressure increase in the pipeline by at least 0.2 $\mathrm{MPa}$ [2]. In this case, the amplitude of the pressure change can reach $2 \mathrm{MPa}$. The stresses arising in this case in the wall of the pipeline can reach values from 60 to $160 \mathrm{MPa}$, depending on the working pressure, diameter and wall thickness of the pipeline.

The basic number of cycles leading to the weakening of the metal of the pipeline wall and the possibility of fatigue cracks appearing in it is a value of $1000-10000$, which is typical for all structures and structures subject to low-cycle effects. For an accurate estimate of the number of cycles in oil pipelines, it is necessary to analyze the data on start-ups and shutdowns of units and switch valves over the past few years. After conducting such an analysis and determining the number of load cycles, we can conclude whether there is a likelihood of critical fatigue damage or not for the pipeline in question.

\subsection{Cyclic loads in main gas pipelines}

The cyclic stresses drops in the walls of the main gas pipelines are caused by pulsations of the pressure of the pumped gas arising due to the technological features of the used gas pumping units. Also, one of the causes of pulsations is a large number of connecting parts installed on the discharge line of the compressor station.

The stress caused by the pulsation of the pumped gas in the wall of the main pipeline can be described using the mathematical expression:

$$
\sigma=\sigma_{1} \sin \left(\omega_{1} t\right)+\sigma_{2} \sin \left(\omega_{2} t+\varphi\right)+\sigma_{m},
$$

where $\sigma_{l}, \sigma_{2^{-}}$amplitude stresses respectively to low and high frequencies;

$\omega_{1}, \omega_{2}, \varphi$ - phase angle between components;

$\sigma_{m}$ - average cycle stress [4].

As a rule, pulsations of the pumping medium have an amplitude within 0.1 MPa, which leads to a slight (30-60 $\mathrm{MPa}$ ) increase in the level of acting stresses in the pipe wall. Such changes in stress cannot cause destruction, since their values do not exceed the yield strength.

In [5-6], the authors performed a calculation according to the method described in [4]. The result of the calculations were the equations of vibration of the acting stresses in the walls of the pipelines. The stress graphs were complex sinusoidal dependences of the level of the acting annular and longitudinal stresses on time. Also, for reference, the value of the yield strength for the studied gas pipeline material was constructed. The maximum amplitude stresses were $65-75 \%$ of the yield strength of the material.

Based on the results of these works, a comparative analysis was carried out, the results of which are shown in table 1 .

Table 1. Comparative analysis of gas pipelines.

\begin{tabular}{|c|c|c|}
\hline $\begin{array}{l}\text { Parameter / } \\
\text { Gas Pipeline }\end{array}$ & $\begin{array}{c}\text { Pipeline laid in } \\
\text { the Far North }\end{array}$ & $\begin{array}{c}\text { Main gas pipeline } \\
\text { "Bovanenkovo- } \\
\text { Ukhta" }\end{array}$ \\
\hline$\sigma_{C S M A X}, \mathrm{MPa}$ & 237 & 363,6 \\
\hline$\sigma_{C S M I N}, \mathrm{MPa}$ & 168,4 & 290,8 \\
\hline $\mathrm{A}_{C S}, \mathrm{MPa}$ & 68,6 & 72,8 \\
\hline$\sigma_{L S M A X}, \mathrm{MPa}$ & 220,1 & 237,7 \\
\hline$\sigma_{L S M I N}, \mathrm{MPa}$ & 199,5 & 215,9 \\
\hline $\mathrm{A}_{L S}, \mathrm{M \Pi a}$ & 20,6 & 21,8 \\
\hline $\mathrm{T}, \mathrm{s}$ & $16870=4,69 \mathrm{~h}$ & $14100=3,91 \mathrm{~h}$ \\
\hline$\sigma_{C S M A X} / \sigma_{0.2}$ & $237 / 345=0,687$ & $363.6 / 520=0.733$ \\
\hline \multicolumn{2}{|c}{}
\end{tabular}

As can be seen from table 1, the key factor influencing the ripple value is the average pressure in the gas pipeline. (for the first gas pipeline, the pressure is 7.4 $\mathrm{MPa}$, for the second, the pressure is $11.8 \mathrm{MPa}$.). Based on the average pressure, the pumping units are backed up. With an increase in unit power of the unit, the value of ripples also increases. For the cycle time, the dependence is inverse, i.e. the time of one cycle is reduced with increasing unit power.

A characteristic feature of the cyclic operation of main gas pipelines is that the most dangerous section that is subject to the influence of cyclic exposure is a section from 2 to $20 \mathrm{~km}$ in length from the compressor station. It is in this area that the effect of gas pulsations is greatest. In the following sections up to the compressor station, the pulsations in the stream are 
damped and the pressure in the pipeline becomes constant.

The main danger factor for multi-cycle fatigue for the main gas pipeline is that pulsations of the medium lead to a slight increase in stresses in the walls of the pipeline. But at the same time, there are various kinds of defects in the walls (dents, scratches, cracks and other corrosion damage), which are stress concentrators. Near these defects, the metal structure changes somewhat and the level of acting stresses can increase several times. This local increase in stress level is taken into account in strength calculations using the stress intensity factor. For this reason, the low-cycle mode of operation is typical for places where defects are present.

Since the number of cycles in gas pipelines is several orders of magnitude higher than in oil pipelines, the main level of danger is an accurate assessment of the stress-strain state in the places where defects are present. After all, even a small crack, which can satisfy the strength condition with a static approach to assessing the condition of the pipeline, can lead to the destruction of the wall under the influence of cyclic loading.

\section{Durable models applicable for trunk pipelines}

When solving the problem of cyclic loads during pipeline operation, the question of how these loads can be taken into account becomes urgent. For an accurate and complete assessment of the technical condition of the pipeline, a complete mathematical model is needed that can take into account cyclic effects, external factors, and the possibility of defects.

The first step in developing such a model is to analyze the loads and impacts acting on the pipeline.

For main gas pipelines, this task is quite simple, since in this case loading has the simplest form - regular loading. To construct a time series of loading, it is required to determine the average value of the effective stress, as well as the maximum and minimum amplitude values.

The second case of cyclic loading, which is characteristic of trunk pipelines, is the case of block loading. For such loads, it is necessary to separate the blocks and assign them serial numbers from 0 to $\mathrm{n}$, and also determine their duration. After that, each n-block is considered separately as regular loading and similar parameters are determined for it.

In the case of an oil pipeline, accidental loading takes place. In this case, it is necessary to accidentally load the form of equivalent block loading in order to be able to use the circuit described in the previous paragraph.

After determining the loading history, it is necessary to determine the approach to the assessment of damage. Since defects are present in pipelines, a model taking into account the presence of local areas that are subject to plastic deformations, while the main part operates in an elastic mode, is necessary for a correct resource estimate.
To solve this problem in an elastic setting, i.e. with respect to stresses $\sigma$ and strains $\varepsilon$, it is necessary to solve the system of equations:

$$
\left\{\begin{array}{l}
\sigma^{e}=\alpha_{\sigma} \sigma \\
\varepsilon^{e}=\alpha_{\sigma} \varepsilon
\end{array}\right.
$$

where $\alpha_{\sigma}$ - theoretical stress concentration coefficient of the considered stress concentrator [7].

When solving this system, it becomes necessary to find the fields of effective stresses and deformation fields. Moreover, when finding the deformation field, one should take into account the fact that the total deformation is the sum of the deformation for the plastic and elastic component. Therefore, to find the desired fields, you can use the Neuber rule. This rule says that for the case of elastic work of the structure, the total energy density is equivalent to the local total energy density that occurs when working in plastic mode. Figure 3 shows a graphical implementation of the Neuber rule.

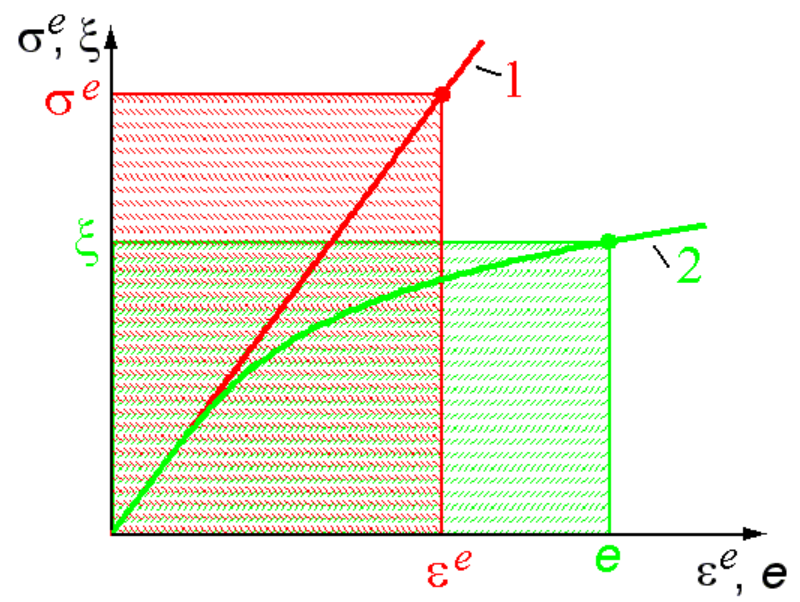

Fig.3. Graphical representation of the distribution of stress and strain fields obeying the Neuber rule.

In the presented diagram, line 1 is a graph of the deformation of an elastic body. Line 2 is a graph of deformation in the inelastic region of the structure.

After analyzing this graph, we can lead Neuber instilled in the following form:

$$
\sigma^{e} \varepsilon^{e}=\xi_{e}
$$

Substituting system (2) into this expression, taking into account the work of metals in the elastic mode for the left side of equation (3), the desired rule can be represented as:

$$
\alpha_{\sigma}^{2} \frac{\sigma^{2}}{E}=\xi e
$$

where $\mathrm{E}$ - modulus of elasticity of the test material.

It is also possible to use the Morrow-Manson equation to describe the long-term strength of pipelines

$$
e_{a}=\frac{\sigma_{f}}{E}(2 N)^{b}+\varepsilon_{f}(2 N)^{c}
$$


where $\sigma_{f}$ - stresses causing destruction of the metal during the half-cycle in the absence of critical plastic deformations;

$\varepsilon_{f}-$ the amount of plastic deformation causing failure during one half-cycle in the absence of elastic deformations;

b - exponent of fatigue strength (exponent of Baskvin),

$\mathrm{c}$ - fatigue viscosity exponent. [8]

The Morrow-Manson equation is applied in the case of cycles symmetric with respect to zero. Since stress cycles in pipelines are not symmetrical, the expression is adjusted taking into account the influence of the average amplitude per cycle on the fatigue properties of the material. The resulting curve can be described using the Smith-Watson-Topper equation:

$$
\xi_{\max } e_{a}=\frac{\sigma_{f}^{2}}{E}(2 N)^{2 b}+\sigma_{f} \varepsilon_{f}(2 N)^{b+c}
$$

The obtained analytical models allow a numerical calculation of the state of the pipeline for any number of cycles and at any time. The disadvantages of the numerical models is that the coefficients used in the formulas are used according to the available long-term strength curves, which may differ from the actual curves corresponding to the operating modes of the pipeline.

\section{Using Artificial Intelligence to Estimate Residual Life}

An alternative to mathematical models can be fatigue testing of samples of pipeline steels that were loaded according to the operating mode of the pipeline. In this case, the fatigue curve will be $95 \%$ consistent with the actual behavior of the pipe metal.

To be able to assess the state of metal damage, it is necessary to determine the change in the properties of the material under the influence of cyclic loads. The investigated properties must comply with non-destructive testing methods used in in-line diagnostics. Typically, such methods are ultrasound and magnetic diagnostics. The studied parameters can be the speed of passage of ultrasonic waves, the magnetic field strength, etc.

In [9], a change in the speed of passage of ultrasonic waves, as well as the magnitude of the gradient of the magnetic field strength versus the number of cycles, for samples of $09 \mathrm{Mn} 2 \mathrm{Si}$ steel exposed to low-cycle loads, was considered. According to the results obtained, with an increase in the number of cycles, both studied parameters decrease.

Since the use of combined flaw detectors is a very common occurrence during diagnostics, it becomes possible to assess the technical condition based on two diagnostic parameters.

In this case, complexity arises since separately from each other there is a dependence of changes in the ultrasonic and magnetic characteristics of the medium. In this case, artificial neural networks can be used to assess the technical condition and predict the residual life.
Artificial neural networks allow you to process heterogeneous dependencies and establish a connection between them. Using ANNs to solve the problem of processing heterogeneous data is much simpler and faster than deriving empirical or semi-empirical dependencies.

Also one of the most important advantages of a neural network is its ability to learn. This means that with an increase in the number of assessments of the technical condition, the accuracy of forecasts will increase.

The program is based on data obtained during cyclic testing of samples loaded in the modes of the corresponding operating modes of the main pipelines. These samples are examined by ultrasonic and magnetic control methods, and the results are entered into the neural network database as a training sample. Based on this sample, the network learns and builds the dependence of the change in diagnostic parameters on the number of cycles.

Thus, the application of such a technique will result in a software product capable of producing an accurate estimate of the current technical condition of the pipeline, as well as forecasting its residual life.

\section{References}

1. Electronic resource: https://gks.ru/storage/mediabank/rus19.pdf

2. Chepurnoy O. V., Myznikov M. O., Beselia D. S., Vansovich K. A., Surikov V. I. Science and Technology of Pipeline Transport of Oil and Oil Products Definition and accounting of loading cycles of a main oil pipeline 3 (19), 23 - 29 (2015)

3. Romashin M.G. Business Journal Neftegaz.RU Calculation of cyclic loads of main oil and oil product pipelines 12 (96), 88-92 (2019)

4. Kharionovsky V.V. Reliability and resource of gas pipeline constructions (Moscow Nedra Publishing House OJSC, 2000)

5. Shchipachev A.M., Lapiga I.R. Bulatov readings: materials of the II International Scientific and Practical Conference (March 31, 2018): in 7 volumes: collection of articles Prediction of the residual resource of oil and gas pipelines by the method of neural network modeling 179-185 (2018)

6. Shchipachev A.M., Lapiga I.R. Business Journal Neftegaz.RU Artificial neural networks for evaluating the residual resource of gas-oil pipelines 12 (96), 46-52 (2019)

7. Feodosiev V.I. Strength of materials (Moscow Nauka, 1967

8. Masing G. Proceedings of the 2nd International Congress of Applied Mechanics Zürich Eigenspannungen und verfestigung beim messing 332-335 (1926)

9. A.M. Shipachov, Y.A. Naumkin, T.F. Musairov Oil and gas business Levels of accumulated metal deterioration evaluation based on neurofuzzy modeling 3, 160-165 (2015) 